\title{
A new near-infrared spectroscopy parameter as marker for patients with migraine
}

\author{
S. Viola $\cdot$ P. Viola $\cdot$ P. Litterio $\cdot$ M. P. Buongarzone $\cdot$ \\ L. Fiorelli
}

(C) Springer-Verlag Italia 2013

\begin{abstract}
Due to the lack of markers for migraine, diagnosis relies entirely on symptomatology. The aim is to evaluate the utility of a new near-infrared spectroscopy parameter as potential marker for patients with episodic migraine with (MA+) and without aura (MA-) during the interictal period of migraine. We studied 10 patients with $\mathrm{MA}+$ (age $39.5 \pm 12.2$ years) and 10 with MA- (age $40.3 \pm 10.2$ years), according to ICHD-II criteria 2004, during the interictal period of migraine and 15 age and sex matched healthy control subjects. The cases and controls were free from vascular risk factors and migraine prophylactic medications. At rest in all the participants, the time delay in millisecond (ms), between the R-wave of an electrocardiogram and the arterial pulse wave of cerebral microcirculation detected by transcranial near-infrared Spectroscopy (R-APWCMtd) on the frontal cortex of both side, was determined. The patients with migraine had a significantly longer R-APWCMtd than the control subjects: the patients with $\mathrm{MA}+:+38.3 \mathrm{~ms}, \quad p<0.0002$; the patients with MA-: $+34.7 \mathrm{~ms}, p<0.0002$, and there was no significant difference between MA+ and MA-, $p=0.71$. We used receiver-operator characteristic (ROC) curve to assess the diagnostic potential of the R-APWCMtd measurement for diagnosis of migraine; we found an ROC area of $0.92, p<0.0001$, sensitivity $77.8 \%$, specificity $100 \%$ and cutoff of $242 \mathrm{~ms}$. Our study seems to indicate that the longer R-APWCMtd is independently associated
\end{abstract}

S. Viola $(\bowtie) \cdot$ P. Litterio - M. P. Buongarzone · L. Fiorelli Department of Neurology, Headache Center,

Via C. De Lellis, 66054 Vasto $(\mathrm{CH})$, Italy

e-mail: stefano.viola@email.it

P. Viola

Emergency Medical Service, S.C. De Lellis Hospital,

Atessa $(\mathrm{CH})$, Italy with a diagnosis of migraine, and may be considered a new marker for migraine, especially in patients with MA+.

Keywords New marker for migraine $\cdot$ Near-infrared spectroscopy $\cdot$ R-APWCMtd $\cdot$ PWV

\section{Introduction}

Migraine is a common chronic disorder affecting up to $14 \%$ of the general population. Due to the lack of markers for migraine, diagnosis relies entirely on symptomatology. The pulse wave velocity (PWV) is the speed at which the forward pressure wave is transmitted from the aorta through the vascular tree. It is calculated by the time taken for the arterial waveform to pass between two points. PWV provides information on the distensibility of the local arterial vessel being studied [1]. Distensibility is inversely related to arterial stiffness [1].

By means of near-infrared Spectroscopy (NIRS), it is possible to detect the arterial pulse wave of cerebral microcirculation (APWCM) on the frontal cortex (Fig. 1) [2]. The aim is to evaluate the utility of a new NIRS parameter as potential marker for patients with migraine with $(\mathrm{MA}+)$ and without aura $(\mathrm{MA}-)$ during the interictal period of migraine.

\section{Methods}

The patients with episodic migraine were consecutively recruited at the outpatient Headache Center of the Neuroscience Department of our hospital from December 2010 to November 2011.

The study was approved by the local ethics committee. 


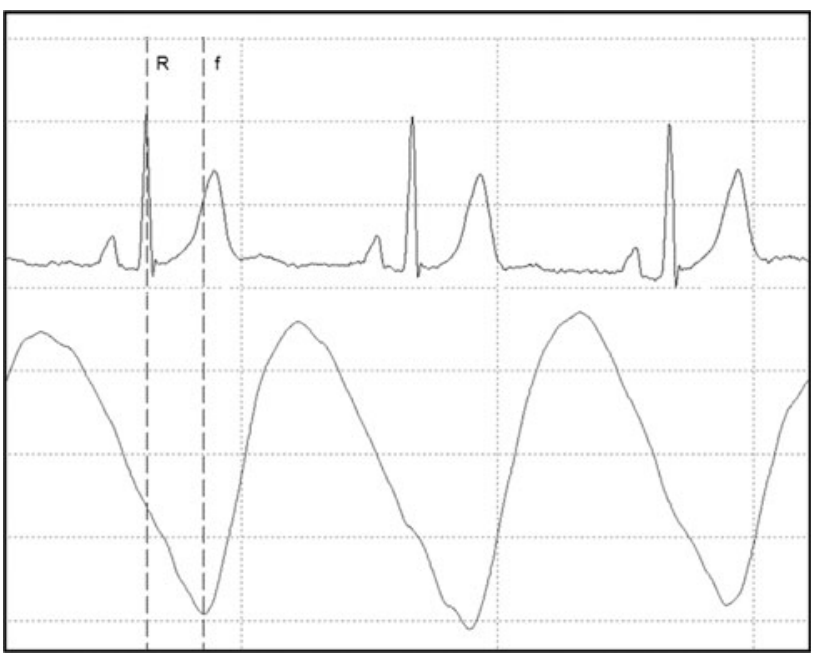

Fig. 1 The time delay ( $218 \mathrm{~ms})$ between the $R$-wave of an electrocardiogram and the foot $(f)$ of arterial pulse wave of cerebral microcirculation (R-APWCMtd) detected by transcranial near-infrared Spectroscopy in a control subject on F2 area

All the patients and control subjects gave their informed consent prior to inclusion in the study.

We studied 10 patients with MA+ (age $39.5 \pm 12.2$ years) and 10 with MA- (age $40.3 \pm 10.2$ years), in according to ICHD-II criteria 2004, during the interictal period of migraine and 15 age, sex and height matched healthy control subjects. We excluded secondary headaches by appropriate laboratory and imaging diagnostic tests. The cases and controls were free from overt cardiovascular events (CVE) including angina pectoris and myocardial infarction, transient ischemic attack (TIA), ischemic stroke, major cardiovascular risk factors (diabetes, hypertension, and hypercholesterolemia) and migraine prophylactic medications. Subjects were asked not to use any inflammatory or analgesic drugs for 3 days before examination. At rest in all the participants, the time delay in millisecond (ms) between the R-wave of an electrocardiogram and the foot of arterial pulse wave of cerebral microcirculation (R-APWCMtd), detected by transcranial NIRS (T-NIRS EVO II, CW system) [2] on the frontal cortex of both side (position F1, F2 of the international 10-20 EEG system), was determined (Fig. 1). Blood pressure was measured on the left arm in the patients and controls.

\section{Statistical analysis}

The association of migraine with the R-APWCMtd values was assessed using independent samples $t$ test; we used receiver-operator characteristic (ROC) curve to assess the diagnostic potential of the R-APWCMtd measurement for diagnosis of migraine.

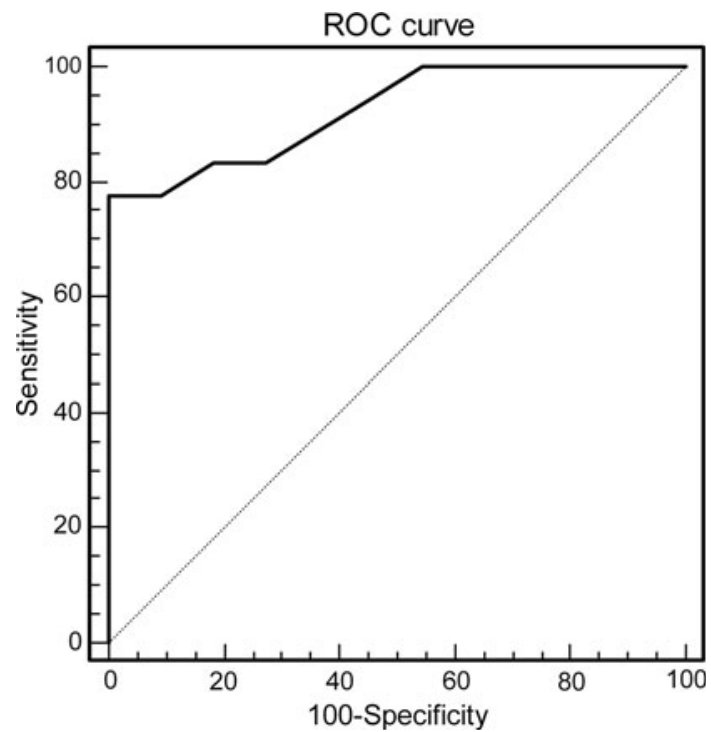

Fig. 2 The ROC curve for R-APWCMtd (frontal cortex) in the migraine patients and controls

\section{Results}

The patients with migraine had a significantly longer R-APWCMtd than the control subjects: the patients with $\mathrm{MA}+:+38.3 \mathrm{~ms}, p<0.0002$; the patients with MA-: $+34.7 \mathrm{~ms}, p<0.0002$. There was no significant difference between the right and left R-APWCMtd of frontal cortex in the migraine patients $p=0.77$ and controls $p=0.70$. There was no significant difference between MA+ and $\mathrm{MA}-, p=0.71$. There was no significant difference in the brachial blood pressure values between the patients and controls $p=0.75$. We found an ROC area of 0.92 , $p<0.0001$, sensitivity $77.8 \%$, specificity $100 \%$ and cutoff of $242 \mathrm{~ms}$ (Fig. 2).

\section{Discussion}

Every heartbeat causes a pulsatile pressure gradient that propagates through the arterial network (aorta, carotid arteries, cerebral microcirculation that includes arterioles, capillaries) and causes local changes in blood flow and volume. The local changes in blood volume are due to the elastic wall of the arterial network. In the microcirculation of cerebral cortex, small local changes in blood volume are still present and are revealed by means of NIRS that we call farterial pulse waves of cerebral microcirculation (APWCM) [2].

In our study, we excluded all subjects with major vascular risk factors, CVE, TIA or ischemic stroke, migraine prophylactic medications and to the subjects were asked not to use any inflammatory or analgesic drugs for 3 days 
before examination. In addition, we assume that intracranial pressure is within the normal range in the patients and controls. These confounding factors may chance the distensibility of cerebral arterial network and therefore R-APWCMtd; in addition, the patients and controls were mached by age, sex and height. This longer R-APWCMtd suggests an increased arterial distensibility (or reduced arterial stiffness) of cerebral arterial network, because the pulsatile pressure gradient propagates more slowly in presence of increased arterial distensibility (according to Bramwell and Hill equation) [1].

A recent study hypothesizes that increased nitric oxide (NO) in the headache-free period is associated with migraine [3]. The increased NO, by reducing the vascular tone altering smooth muscle tone, leads to vasodilatation of cerebral arteries and could increase the arterial distensibility of cerebral vessels [4] that we found in our migraine patients. In addition, serum elastase activity is elevated in migraine [5]. This enzyme is involved in extracellular matrix degradation [5]. The higher level of extracellular matrix degradation may explain the increased arterial distensibility of cerebral arterial network in our migraine patients. The increased arterial distensibility of cerebral vessels, reducing the resistance of cerebral arteries, increases the blood flow pressure in cerebral arterioles (resistance vessels). By means of cerebral vascular autoregulation, we suppose that the increased blood flow pressure leads to a mild vasoconstriction of cerebral arterioles in migraine patients. Finally, the increased arterial distensibility of cerebral vessels may alter pulse wave reflection, shear stress on endothelial cells and endothelial function. The findings of our study suggest altered biomechanical properties of the wall of cerebral arterial network in migraine patients. In addition, the altered biomechanical properties of the wall of cerebral arterial network provides one potential physiological explanation for the increased risk of cervical artery dissection in migraine and increased prevalence of migraine in Marfan Syndrome [6].
In our study, ROC curve is used to determine if the R-APWCMtd measurement provides a basis for distinguishing controls from subjects with migraine. This analysis, showing the ROC area greater than 0.7 and closest to 1.0, suggests that the longer R-APWCMtd might have the greatest diagnostic potential. R-APWCMtd above the cutoff value appears closely associated with the diagnosis of migraine.

In conclusion, in our migraine patients, by means of NIRS and an electrocardiogram, safe, repeatable and noninvasive technologies, our study seems to indicate that the longer R-APWCMtd is independently associated with a diagnosis of migraine. The longer R-APWCMtd may be considered a new marker for migraine, especially in patients with MA+.

Finally, it is possible to speculate that therapeutic approaches, aiming at correcting R-APWCMtd, may also improve the migraine.

The sample size was relatively small, and further confirmation by a study with a larger population is needed.

Conflict of interest The authors certify that there is no actual or potential conflict of interest in relation to this article.

\section{References}

1. Mackenzie IS, Wilkinson IB, Cockcroft JR (2002) Assessment of arterial stiffness in clinical practice. QJM 95:67-74

2. Viola S, Viola P, Litterio P, Buongarzone MP, Fiorelli L (2012) Correlation between the arterial pulse wave of the cerebral microcirculation and CBF during breath holding and hyperventilation in human. Clin Neurophysiol 123:1931-1936

3. Gruber HJ, Bernecker C, Lechner A et al (2010) Increased nitric oxide stress is associated with migraine. Cephalalgia 30:486-492

4. Zieman SJ, Melenovsky V, Kass DA (2005) Mechanisms, pathophysiology, and therapy of arterial stiffness. Arterioscler Thromb Vasc Biol 25:932-943

5. Tzourio C, El Armani M, Robert L, Alpérovitch A (2000) Serum elastase activity is elevated in migraine. Ann Neurol 47:648-651

6. Koppen H, Vis JC, Gooiker DJ et al (2012) Aortic root pathology in Marfan syndrome increases the risk of migraine with aura. Cephalalgia 32:467-472 